\title{
Fuzzy Positive Primitive Formulas
}

\author{
Pilar Dellunde \\ 1 Universitat Autònoma de Barcelona, \\ pilar.dellunde@uab.cat \\ 2 Barcelona Graduate School of Mathematics, \\ 3 Artificial Intelligence Research Institute IIIA-CSIC
}

\begin{abstract}
Can non-classical logic contribute to the analysis of complexity in computer science? In this paper, we give an step towards the solution of this open problem, taking a logical model-theoretic approach to the analysis of complexity in fuzzy constraint satisfaction. We study fuzzy positive-primitive sentences, and we present an algebraic characterization of classes axiomatized by these kind of sentences in terms of homomorphisms and finite direct products. The ultimate goal is to study the expressiveness and reasoning mechanisms of non-classical languages, with respect to constraint satisfaction problems and, in general, in modelling decision scenarios.
\end{abstract}

Keywords: fuzzy constraint satisfaction, preference modeling, fuzzy logics, model theory.

\section{Introduction}

Can non-classical logic contribute to the analysis of complexity in computer science? The motivation to answer this question comes, in the first place, from the reading of [22], where some open problems were proposed by the authors about the relationship between fuzzy logic and valued constraint satisfaction. Io our opinion, a research oriented to find a non-classical logical approach to complexity, should address, at least, the following three issues:

1. Show that there is a good trade-off between algebra and logic in the relevant fragments.

2. Identify which problems in complexity theory are naturally expressed as questions about the expressive power of the non-classical logic.

3. Prove that these complexity problems are not better addressed in other known logical formalisms.

Of course, all these issues are interrelated. To evaluate the trade-off between algebra and logic, it is important to identify which are the relevant fragments of the non-classical logic where the complexity problems have to be expressed; and to prove the relevancy of the fragments, a comparative study of different logical formalisms with respect to their expressive power has to be performed. 
Revisiting the role of non-classical logics in computer science, has to be done both, in general terms, trying to find a uniform approach, but also focusing on particular classes of problems naturally addressed for some non-classical logics, as it is the case of this paper, where we contribute to the model-theoretic analysis of fuzzy constraint satisfaction using predicate fuzzy logics.

Constraint-based modeling has become a central research area in computational social choice, and in particular in preference modeling, where preferences can be seen as soft constraints [18]. Different soft constraint formalisms can be found in the literature, some prominent examples are fuzzy constraint satisfaction ([10], [25]), possibilistic [19], probabilistic [12], and weighted [26]. More recently, the semiring-based and the valued constraint general framework have been introduced ([3] and [26], respectively), and previous formalisms can conveniently be regarded as instances of semiring-based or valued soft constraints. For a general reference to the different soft-constraint formalisms in preference modeling see [21] and [18].

The classical constraint satisfaction problem (CSP) has been proved to have strong connections with various problems in database theory and classical finitemodel theory [15], where CSP can be rephrased as a homomorphism problem, a conjunctive-query evaluation problem, or a join-evaluation problem. Some problems in complexity theory are naturally expressed as questions about the expressive power of certain classical logics. With the plurality of valued structures involved in soft contraint problems, it is a natural question to ask, for the relationship between valued CSP and non-classical logical formalisms. In particular, as pointed out in [22], with mathematical fuzzy logic (MFL). Only in recent times, model theory of predicate fuzzy logics has been developed as a subarea of MFL (see for instance [5] or [9]), leaving the important area of fuzzy finite-model theory yet unexplored.

Considering a general semantics for MFL, a plethora of left continuous tnorms can be defined, going far beyond of the minimum t-norm in the interval $[0,1]$ of the reals, most commonly used in fuzzy CSP (FCSP). Nevertheless, as pointed already in some earlier works (see for instance [25] or [27]) the minimum is the only total order semiring operator that is idempotent (see also [26]), and its drowning effect limits the application of FCSP to specific contexts (for a recent example of the application of fuzzy constraints in compact preference representation see [20]). t-norms in general are not good as aggregation operators, but our research do not want to focus only in aggregation, we would like rather to explore the logical properties of fuzzy languages, their expressiveness, and reasoning mechanisms with respect to constraint satisfaction problems and, in general, in modeling decision scenarios [2].

Positive-primitive formulas are one of the key elements in the logical study of classical CSP (see for instance [15]). The original contribution of the article is the mathematical proof of an axiomatization theorem for primitive-positive theories. The proof uses specific techniques of model theory and algebra in the fuzzy context, and it is included in section 4. Some preliminaries on FCSP, and predicate fuzzy logics needed for the theorem are introduced in sections 2 and 
3. A discussion section at the end of the paper presents some ideas for future work.

\section{Preliminaries}

Fuzzy CSP The valued structure most commonly used in the literature of fuzzy constraint satisfaction is the standard Gödel algebra, that has as domain the $[0,1]$ interval of the real numbers, and as t-norm the minimum. In this paper, we will work with MTL-algebras, which constitute the set of truth-values where sentences of predicate fuzzy logic are evaluated. We focus on finite MTL-algebras, but the results can be extended to the case where the valued structure is, for instance, the infinite standard Gödel or Łukasiewicz algebra. The domains of the finite MTL-algebras we consider are not necessarily totally ordered, allowing to represent some types of non-linear preferences.

MTL-algebras are defined as bounded integral commutative residuated lattices $(A, \sqcap, \sqcup, *, \Rightarrow, 0,1)$, where $\sqcap$ and $\sqcup$ are respectively the lattice meet and join operations, $*$ is a left-continuous t-norm, and $(\Rightarrow, *)$ is a residuated pair (for an exhaustive exposition of MTL-algebras we refer to [11]).

Definition 1. Let $\mathbf{A}$ be a MTL-algebra, $D$ a set, and $k$ a natural number. It is said that $R$ is a $k$-ary fuzzy relation on $D$, if $R: D^{k} \rightarrow A$ is a function evaluated in $A$.

Definition 2. An instance $\mathcal{I}$ of fuzzy constraint satisfaction is a triple $(V, D, C)$, where

- $V$ is a set of variables;

- $D$ is a set of values, referred to as the domain;

- $C$ is a collection of constraints $C_{1}, \ldots, C_{q}$, where each constraint $C_{i}$ is a pair $\left(\bar{x}, R^{\mathcal{I}}\right)$, where $R^{\mathcal{I}}$ is a $k$-ary fuzzy relation on $D$, for some natural number $k \geq 1$, and $\bar{x}$ is a $k$-tuple over $V$, referred to as the scope of the constraint.

Given an instance $\mathcal{I}$ of fuzzy constraint satisfaction with set of constraints $C=\left\{\left(\bar{x}_{1}, R_{1}^{\mathcal{I}}\right), \ldots,\left(\bar{x}_{n}, R_{n}^{\mathcal{I}}\right)\right\}$, and a $k$-tuple $\bar{d} \in D$, we say that $R_{i}^{\mathcal{I}}(\bar{d})$ is the degree of satisfaction of $\bar{d} \in D$ of constraint $\left(\bar{x}_{i}, R_{i}^{\mathcal{I}}\right)$, and that $R_{1}^{\mathcal{I}}(\bar{d}) * \cdots * R_{n}^{\mathcal{I}}(\bar{d})$ is the degree of joint satisfaction of the constraints, where $*$ is the t-norm of the algebra $\mathbf{A}$. For the sake of clarity, we have restricted the definition to the case where the degree of joint satisfaction is calculated only by means of the t-norm * in the standard way, but other functions could have been introduced using as base both $*$ and the min.

The Fuzzy Constraint Satisfaction Problem is to find an optimal solution, in the sense of maximazing the degree of joint satisfaction of the constraints. Related to this central problem there is a variety of other problems that it is possible to formulate using the graded nature of fuzzy constraints, for instance, we can ask if there is a $k$-tuple $\bar{d} \in D$ such that the degree of joint satisfaction is greater or lower than a given threshold. 
Predicate Fuzzy Logics Given an instance $\mathcal{I}$ of fuzzy constraint satisfaction with set of constraints $C=\left\{\left(\bar{x}_{1}, R_{1}^{\mathcal{I}}\right), \ldots,\left(\bar{x}_{n}, R_{n}^{\mathcal{I}}\right)\right\}$, we can associate to $\mathcal{I}$ a fuzzy relational $\mathbf{A}$-structure $\mathcal{I}=\left(D, R_{1}^{\mathcal{I}}, \ldots, R_{n}^{\mathcal{I}}\right)$, and study its properties using model theory of predicate fuzzy logics. Now we present the syntax and semantics of the minimal predicate fuzzy logic MTL $\forall^{m}$, the predicate extension of the leftcontinuous t-norm based logic MTL introduced in [11], and we refer to [6, Ch.1] for a complete and extensive presentation of MTL $\forall^{m}$.

Definition 3 (Syntax of Predicate Languages). A predicate language $\mathcal{P}$ is a triple $\left\langle\right.$ Pred $_{\mathcal{P}}$, Func $\left._{\mathcal{P}}, A r_{\mathcal{P}}\right\rangle$, where Pred $_{\mathcal{P}}$ is a nonempty set of predicate symbols, Func $c_{\mathcal{P}}$ is a set of function symbols (disjoint from Pred $d_{\mathcal{P}}$ ), and $A r_{\mathcal{P}}$ represents the arity function, which assigns a natural number to each predicate symbol or function symbol. We call this natural number the arity of the symbol. The predicate symbols with arity zero are called truth constants, while the function symbols whose arity is zero are named individual constants.

The set of $\mathcal{P}$-terms, $\mathcal{P}$-formulas and the notions of free occurrence of a variable, open formula, substitutability and sentence are defined as in classical predicate logic. We asume that the equality symbol $\approx$ of the language is interpreted in every structure as the crisp identity. Notice that, in the language we have introduced there are also function symbols. The results we present in this paper hold also for arbritrary languages, and for this reason we have presented a general proof, that could be used in further applications of pp-definability in non-relational structures, not necessarily related to FCSP.

Definition 4. We introduce an axiomatic system for the predicate logic MTL $\forall^{m}$ :

(P) Instances of the axioms of the propositional logic MTL.

$(\forall 1) \quad(\forall x) \varphi(x) \rightarrow \varphi(t)$, where the term $t$ is substitutable for $x$ in $\varphi$.

( $\exists 1) \quad \varphi(t) \rightarrow(\exists x) \varphi(x)$, where the term $t$ is substitutable for $x$ in $\varphi$.

$(\forall 2) \quad(\forall x)(\xi \rightarrow \varphi) \rightarrow(\xi \rightarrow(\forall x) \varphi(x))$, where $x$ is not free in $\xi$.

$(\exists 2) \quad(\forall x)(\varphi \rightarrow \xi) \rightarrow((\exists x) \varphi \rightarrow \xi)$, where $x$ is not free in $\xi$.

The deduction rules of MTL $\forall^{m}$ are those of MTL and the rule of generalization: from $\varphi$ infer $(\forall x) \varphi$. The definitions of proof and provability are analogous to the classical ones. A set of formulas $\Phi$ is consistent, if $\Phi \nvdash \overline{0}$.

From now on we fix a finite MTL-algebra A and consider only structures over this algebra.

Definition 5 (Semantics of Predicate Fuzzy Logics). Consider a predicate language $\mathcal{P}=\left\langle\right.$ Pred $_{\mathcal{P}}, F$ unc $\left._{\mathcal{P}}, A r_{\mathcal{P}}\right\rangle$. We define an $\mathbf{M}$-structure $\mathbf{M}$ for $\mathcal{P}$ as a triple $\left\langle M,\left(P_{\mathbf{M}}\right)_{P \in \text { Pred }},\left(F_{\mathbf{M}}\right)_{F \in F u n c}\right\rangle$, where $M$ is a nonempty domain, $P_{\mathbf{M}}$ is an $n$-ary fuzzy relation for each $n$-ary predicate symbol, identified with an element of $\boldsymbol{A}$, if $n=0$; and $F_{\mathbf{M}}$ is a function from $M^{n}$ to $M$, identified with an element of $M$, if $n=0$.

As usual, if $\mathbf{M}$ is an $\mathbf{A}$-structure for $\mathcal{P}$, an $\mathbf{M}$-evaluation of the object variables is a mapping $v$ assigning to each object variable an element of $M$. The 
set of all object variables is denoted by Var. If $v$ is an $\mathbf{M}$-evaluation, $x \in V$ Var and $a \in M$, we denote by $v[x \mapsto a]$ the $\mathbf{M}$-evaluation so that $v[x \mapsto a](x)=a$ and $v[x \mapsto a](y)=v(y)$ for $y$ an object variable such that $y \neq x$. If $\mathbf{M}$ is an $\mathbf{M}$-structure and $v$ is an $\mathbf{M}$-evaluation, we define the values of terms, and the truth values of formulas in $M$ for an evaluation $v$ recursively as follows:

$$
\begin{aligned}
& \|x\|_{\mathbf{M}, v}=v(x) ; \\
& \left\|F\left(t_{1}, \ldots, t_{n}\right)\right\|_{\mathbf{M}, v}=F_{\mathbf{M}}\left(\left\|t_{1}\right\|_{\mathbf{M}, v}, \ldots,\left\|t_{n}\right\|_{\mathbf{M}, v}\right), \text { for } F \in F u n c ; \\
& \left\|P\left(t_{1}, \ldots, t_{n}\right)\right\| \|_{\mathbf{M}, v}=P_{\mathbf{M}}\left(\left\|t_{1}\right\|_{\mathbf{M}, v}, \ldots,\left\|t_{n}\right\|_{\mathbf{M}, v}\right), \text { for } P \in \text { Pred; } \\
& \left\|\lambda\left(\varphi_{1}, \ldots, \varphi_{n}\right)\right\|_{\mathbf{M}, v}=\lambda_{\mathbf{A}}\left(\left\|\varphi_{1}\right\|_{\mathbf{M}, v}, \ldots,\left\|\varphi_{n}\right\|_{\mathbf{M}, v}\right), \text { for every connective } \lambda ; \\
& \|(\forall x) \varphi\|_{\mathbf{M}, v}=\inf \left\{\|\varphi\|_{\mathbf{M}, v[x \rightarrow a]} \mid a \in M\right\} ; \\
& \|(\exists x) \varphi\|_{\mathbf{M}, v}=\sup \left\{\|\varphi\|_{\mathbf{M}, v[x \rightarrow a]} \mid a \in M\right\} .
\end{aligned}
$$

We assume that the language has an equality symbol $\approx$, interpreted as a crisp identity. We denote by $\|\varphi\|_{\mathbf{M}}=1$ the fact that $\|\varphi\|_{\mathbf{M}, v}=1$ for all $\mathbf{M}$-evaluation $v$; and given a set of sentences $\Phi$, we say that $\mathbf{M}$ is a model of $\Phi$, if for every $\varphi \in \Phi,\|\varphi\|_{\mathbf{M}}=1$. We denote by $\operatorname{Mod}(\Phi)$ the set of models of $\Phi$, and by $\operatorname{Th}(\mathbf{M})$, the theory of $\mathbf{M}$, that is, the set of sentences evaluated 1 in $\mathbf{M}$. We say that two models are elementary equivalent, if they have the same theory.

Structures over a fixed finite MTL-algebra Since we work with structures over a fixed finite MTL-algebra, the infimum and the supremum in Definition 5 always exist, and they coincide with the minimum and maximum. There are two important properties that all the structures over a finite MTL-algebra have, and that we will use throughout this article. The first one is that they are existentially witnessed: given a $\mathbf{A}$-structure $\mathbf{M}$, we say that $\mathbf{M}$ is $\exists$-witnessed if it satisfies the following property: for every formula of the form $(\exists \bar{x}) \psi(\bar{x})$, there are $\bar{d} \in M$ such that $\|(\exists \bar{x}) \psi(\bar{x})\|_{\mathbf{M}}=\|\psi(\bar{d})\|_{\mathbf{M}}$.

The second property is compactness, both for satisfiabilty and consequence (the proof can be found in [8, Th. 4.4]). Remark that, in fuzzy logic it is not always the case, for instance the product predicate logic is neither satisfiability nor consequence compact with respect to its standard algebra. Given a set of sentences $\Sigma$, and a sentence $\phi$, we denote by $\Sigma \models_{\mathbf{A}} \phi$ the fact that every $\mathbf{A}$ model of $\Sigma$ is also an A-model of $\phi$.

Theorem 1 ( $A$-compactness). For every set of sentences $\Sigma$ and sentence $\phi$, the following holds:

1. [Satisfiability] If for every finite subset $\Sigma_{0} \subseteq \Sigma, \Sigma_{0}$ has an $\mathbf{A}$-model, then $\Sigma$ has also an A-model.

2. [Consequence] If $\Sigma \models_{\mathbf{A}} \phi$, then there is a finite subset $\Sigma_{0} \subseteq \Sigma$ such that $\Sigma_{0}=_{\mathbf{A}} \phi$.

From now on we will refer to A-structures simply as structures, because all the structures we consider will be over the same algebra. 


\section{$3 \quad$ Fuzzy Positive-Primitive Formulas}

Let $\mathcal{I}$ be an instance of fuzzy constraint satisfaction and $\mathcal{I}=\left(D, R_{1}^{\mathcal{I}}, \ldots, R_{n}^{\mathcal{I}}\right)$ its associated fuzzy relational structure. In logical terms, the FCSP can be formulated as the problem of finding a tuple $\bar{d}$ such that

$$
\left\|R_{1}(\bar{d}) \& \cdots \& R_{n}(\bar{d})\right\|_{\mathcal{I}}=\left\|(\exists \bar{x})\left(R_{1}(\bar{x}) \& \cdots \& R_{n}(\bar{x})\right)\right\|_{\mathcal{I}}
$$

where $\&$ is the strong conjunction interpreted in $\mathcal{I}$ as the t-norm. The formulas that allow us to give a logical expression of the FSCP are called fuzzy positiveprimitive and are the object of study of this section. In particular, we show that homomorphisms and direct products preserve fuzzy positive-primitive formulas. For a general reference of the classical positive-primitive fragment see [14].

Definition 6 (Fuzzy Positive-Primitive Formula). Given a predicate language $\mathcal{P}$, and a $\mathcal{P}$-formula $\phi$, it is said that $\phi$ is fuzzy positive-primitive, if $\phi$ is of the form $(\exists \bar{x}) \psi$, where $\psi$ is a quantifier-free formula built from atomic formulas by using only the connectives $\wedge$ and \&.

For the sake of simplicity, from now on we will refer to fuzzy positive-primitive formulas, simply as pp-formulas. Remark that both conjunctions, strong and weak, can appear in pp-formulas, allowing different combinations of these connectives for expressing the degree of joint satisfaction of a set of constraints. Let us recall now the definition of homomorphism introduced in [9] as a generalization of the notion of classical homomorphism.

Definition 7 (Homomorphism). Let $\mathcal{P}$ be a predicate language, $\mathbf{M}$ and $\mathbf{N}$ be two $\mathcal{P}$-structures and $g$ a mapping from $M$ to $N$. We say that $g$ is a homomorphism from $\mathbf{M}$ into $\mathbf{N}$ if and only if

1. For every $n$-ary function symbol $F \in \mathcal{P}$, and $d_{1}, \ldots, d_{n} \in M$,

$$
g\left(F_{\mathbf{M}}\left(d_{1}, \ldots, d_{n}\right)\right)=F_{\mathbf{N}}\left(g\left(d_{1}\right), \ldots, g\left(d_{n}\right)\right) .
$$

2. For every $n$-ary predicate symbol $P \in \mathcal{P}$, and $d_{1}, \ldots, d_{n} \in M$,

$$
\text { if }\left\|P\left(d_{1}, \ldots, d_{n}\right)\right\|_{\mathbf{M}}=1 \text {, then }\left\|P\left(g\left(d_{1}\right), \ldots, g\left(d_{n}\right)\right)\right\|_{\mathbf{N}}=1 \text {. }
$$

Moreover, we say that $g$ is an embedding, if $g$ is one-to-one, and that $g$ is an isomorphism, if $g$ is a surjective embedding.

In the following lemma we prove that pp-formulas are preserved by homomorphisms.

Lemma 1. Let $\mathcal{P}$ be a predicate language, $\mathbf{M}$ and $\mathbf{N}$ be two $\mathcal{P}$-structures, $g$ a homomorphism from $\mathbf{M}$ into $\mathbf{N}$, and $\phi$ a positive-primitive $\mathcal{P}$-formula. Then, for every $d_{1}, \ldots, d_{n} \in M$,

$$
\text { if }\left\|\phi\left(d_{1}, \ldots, d_{n}\right)\right\|_{\mathbf{M}}=1 \text {, then }\left\|\phi\left(g\left(d_{1}\right), \ldots, g\left(d_{n}\right)\right)\right\|_{\mathbf{N}}=1 .
$$


Proof. By induction on the complexity of $\phi$.

Atomic step. Let $\phi$ be an atomic formula of the form $P\left(t_{1} \ldots, t_{k}\right)$, where $P \in \mathcal{P}$ is a predicate symbol, and $t_{1} \ldots, t_{k}$ are $\mathcal{P}$-terms. Since $g$ is a homomorphism, we have that, in general, for every $\mathcal{P}$-term $t$, and $d_{1}, \ldots, d_{n} \in M$, $g\left(t_{\mathbf{M}}\left(d_{1}, \ldots, d_{n}\right)\right)=t_{\mathbf{N}}\left(g\left(d_{1}\right), \ldots, g\left(d_{n}\right)\right)$ and thus

$$
\begin{aligned}
& \left\|P\left(t_{1} \ldots, t_{k}\right)\left(d_{1}, \ldots, d_{n}\right)\right\|_{\mathbf{M}}=1 \Rightarrow \\
& \left\|P\left(t_{1} \mathbf{M}\left(d_{1}, \ldots, d_{n}\right), \ldots, t_{k} \mathbf{M}\left(d_{1}, \ldots, d_{n}\right)\right)\right\|_{\mathbf{M}}=1 \Rightarrow \\
& \left\|P\left(g\left(t_{1} \mathbf{M}\left(d_{1}, \ldots, d_{n}\right)\right), \ldots, g\left(t_{k} \mathbf{M}\left(d_{1}, \ldots, d_{n}\right)\right)\right)\right\|_{\mathbf{N}}=1 \Rightarrow \\
& \| P\left(t_{1} \mathbf{N}\left(g\left(d_{1}\right), \ldots, g\left(d_{n}\right)\right), \ldots, t_{k} \mathbf{N}\left(g\left(d_{1}\right), \ldots, g\left(d_{n}\right)\right) \|_{\mathbf{N}}=1 \Rightarrow\right. \\
& \left\|P\left(t_{1} \ldots, t_{k}\right)\left(g\left(d_{1}\right), \ldots, g\left(d_{n}\right)\right)\right\|_{\mathbf{N}}=1 .
\end{aligned}
$$

Quantifier-free. Assume inductively that the property holds for $\psi$ and for $\chi$, then we have:

$$
\begin{aligned}
& 1=\left\|\psi \& \chi\left(d_{1}, \ldots, d_{n}\right)\right\|_{\mathbf{M}}=\left\|\psi\left(d_{1}, \ldots, d_{n}\right)\right\|_{\mathbf{M}} *\left\|\chi\left(d_{1}, \ldots, d_{n}\right)\right\|_{\mathbf{M}} \Rightarrow \\
& \left\|\psi\left(d_{1}, \ldots, d_{n}\right)\right\| \|_{\mathbf{M}}=1 \text { and }\left\|\chi\left(d_{1}, \ldots, d_{n}\right)\right\|_{\mathbf{M}}=1 \Rightarrow \\
& \left\|\psi\left(g\left(d_{1}\right), \ldots, g\left(d_{n}\right)\right)\right\|_{\mathbf{N}}=1 \text { and }\left\|\chi\left(g\left(d_{1}\right), \ldots, g\left(d_{n}\right)\right)\right\|_{\mathbf{N}}=1 \Rightarrow \\
& \left\|\psi\left(g\left(d_{1}\right), \ldots, g\left(d_{n}\right)\right)\right\|_{\mathbf{N}} *\left\|\chi\left(g\left(d_{1}\right), \ldots, g\left(d_{n}\right)\right)\right\|_{\mathbf{N}}=1 \Rightarrow \\
& \left\|\psi \& \chi\left(g\left(d_{1}\right), \ldots, g\left(d_{n}\right)\right)\right\|_{\mathbf{N}}=1 .
\end{aligned}
$$

Observe that the same argument holds for the weak conjunction $\wedge$.

Existential step. Assume inductively that the property holds for $\psi(x)$. Since $\mathbf{M}$ is an $\exists$-witnessed structure, we have that for some $e \in M$,

$$
\left\|(\exists x) \psi\left(x, d_{1}, \ldots, d_{n}\right)\right\|_{\mathbf{M}}=\left\|\psi\left(e, d_{1}, \ldots, d_{n}\right)\right\|_{\mathbf{M}}
$$

Thus, if $\left\|(\exists x) \psi\left(x, d_{1}, \ldots, d_{n}\right)\right\|_{\mathbf{M}}=1$, then $\left\|\psi\left(e, d_{1}, \ldots, d_{n}\right)\right\|_{\mathbf{M}}=1$ and, by inductive hypothesis,

$$
1=\left\|\psi\left(g(e), g\left(d_{1}\right), \ldots, g\left(d_{n}\right)\right)\right\|_{\mathbf{N}} \leq\left\|(\exists x) \psi\left(x, g\left(d_{1}\right), \ldots, g\left(d_{n}\right)\right)\right\|_{\mathbf{N}} .
$$

Now let us introduce the notion of direct product. Unlike other definitions introduced in the literature, for instance in [22], we work in products over the same algebra A. Notice that the product is well-defined because the algebra is finite.

Definition 8 (A-direct product). Let $\mathcal{P}$ be a predicate language, I a nonempty set, and for every $i \in I, \mathbf{M}_{i}$ a $\mathcal{P}$-structure. The direct product of the family $\left\{\mathbf{M}_{i}: i \in I\right\}$, denoted by $\prod_{i \in I} \mathbf{M}_{i}$, is the structure that has as domain the usual classical direct product, and the usual classical interpretation for constants and function symbols, and for every $n$-adic predicate symbol $P \in \mathcal{P}$, and tuples of elements $\overline{d_{1}}, \ldots, \overline{d_{n}}$ of $\prod_{i \in I} M_{i}$,

$$
P_{\prod_{i \in I} \mathbf{M}_{i}}\left(\overline{d_{1}}, \ldots, \overline{d_{n}}\right)=\min \left\{P_{\mathbf{M}_{i}}\left(\overline{d_{1}}(i), \ldots, \overline{d_{n}}(i)\right): i \in I\right\}
$$


Notice that, so defined, the $i$-projection of the direct product onto $\mathbf{M}_{i}$ is a homomorphism, and thus, by Lemma 1, preserves pp-formulas. We will use this fact later in the proof of the axiomatization theorem. In the following lemma we prove that pp-formulas are preserved by direct products.

Lemma 2. Let $\mathcal{P}$ be a predicate language, $I$ a nonempty set, and for every $i \in I, \mathbf{M}_{i}$ a $\mathcal{P}$-structure. Assume that $\phi$ is a positive-primitive $\mathcal{P}$-formula, and $\overline{d_{1}}, \ldots, \overline{d_{n}}$ are tuples of elements of $\prod_{i \in I} M_{i}$. Then the following holds: if for every $i \in I,\left\|\phi\left(\overline{d_{1}}(i), \ldots, \overline{d_{n}}(i)\right)\right\|_{\mathbf{M}_{i}}=1$, then $\left\|\phi\left(\overline{d_{1}}, \ldots, \overline{d_{n}}\right)\right\|_{\prod_{i \in I} \mathbf{M}_{i}}=1$.

Proof. By induction on the complexity of $\phi$. The proof of the atomic and quantifierfree step is analogous to the corresponding proof in Lemma 1, by using the fact that for every $\mathcal{P}$-term $t$,

$$
{ }^{t} \prod_{i \in I} \mathbf{M}_{i}\left(\overline{d_{1}}, \ldots, \overline{d_{n}}\right)=\left(t_{\mathbf{M}_{\mathbf{i}}}\left(\overline{d_{1}}(i), \ldots, \overline{d_{n}}(i)\right): i \in I\right)
$$

For the existential step, assume inductively that the property holds for $\psi(x)$. If for every $i \in I,\left\|(\exists x) \psi\left(x, \overline{d_{1}}(i), \ldots, \overline{d_{n}}(i)\right)\right\|_{\mathbf{M}_{i}}=1$, since the structures are $\exists$-witnessed, then for every $i \in I$, there is $\bar{e}(i) \in M_{i}$ such that

$$
\left\|\psi\left(\bar{e}(i), \overline{d_{1}}(i), \ldots, \overline{d_{n}}(i)\right)\right\|_{\mathbf{M}_{i}}=1
$$

Then, by using the inductive hypothesis,

$$
1=\left\|\psi\left(\bar{e}, \overline{d_{1}}, \ldots, \overline{d_{n}}\right)\right\|_{\prod_{i \in I} \mathbf{M}_{i}} \leq\left\|(\exists x) \psi\left(x, \overline{d_{1}}(i), \ldots, \overline{d_{n}}(i)\right)\right\|_{\prod_{i \in I} \mathbf{M}_{i}} .
$$

\section{Fuzzy Positive-Primitive Sets of Axioms}

Axiomatization theorems provide a correspondence between sintactic and semantic notions in logic. Diagrams are the building blocks that, glued with compactness, allow us to build extensions of structures, and prove these axiomatization theorems. Let us thus to introduce the method of diagrams in this fuzzy setting in order to characterize homomorphisms, and prove an equivalent condition to the preservation of pp-formulas between structures.

Definition 9. Let $\mathcal{P}$ be a predicate language, and $\mathbf{M}$ a $\mathcal{P}$-structure. The expansion of the language $\mathcal{P}$ by adding an individual constant symbol $c_{m}$ for every $m \in M$, is denoted by $\mathcal{P}^{M}$; and the expansion of the structure $\mathbf{M}$ to $\mathcal{P}^{M}$ is denoted by $\mathbf{M}^{\sharp}$, where for every $m \in M,\left(c_{m}\right)_{\mathbf{M}^{\sharp}}=m$.

Definition 10. Let $\mathcal{P}$ be a predicate language. For every $\mathcal{P}$-structure $\mathbf{M}$ we define $\operatorname{Diag}(\mathbf{M})$ as the set of atomic $\mathcal{P}^{M}$-sentences $\sigma$ such that $\|\sigma\|_{\mathbf{M}^{\sharp}}=1$.

Following the same lines of the proof of [7, Prop. 32], we can obtain this characterization of homomorphisms in terms of diagrams.

Corollary 1. Let $\mathcal{P}$ be a predicate language and $\mathbf{M}$ and $\mathbf{N}$ be two $\mathcal{P}$-structures. The following are equivalent: 
1. There is an expansion of $\mathbf{N}$ that is a model of $\operatorname{Diag}(\mathbf{M})$.

2. There is a homomorphism $g: M \rightarrow N$ from $\mathbf{M}$ into $\mathbf{N}$.

Notice that, since the $\operatorname{Diag}(\mathbf{M})$ contains equalities but not inequalities, the obtained homomorphism does not need to be an embedding. Now we present a characterization in terms of extensions, of when two structures preserve ppformulas.

Proposition 1. Let $\mathcal{P}$ be a predicate language, and $\mathbf{M}$ and $\mathbf{N}$ be two $\mathcal{P}$-structures. Then, every pp-sentence which is evaluated 1 in $\boldsymbol{M}$, is also evaluated 1 in $\boldsymbol{N}$ if and only if there is a $\mathcal{P}$-structure $\mathbf{L}$, elementary equivalent to $\mathbf{N}$, and a homomorphism $g$ from $\mathbf{M}$ into $\mathbf{L}$.

Proof. First we show that $\operatorname{Diag}(\mathbf{M}) \cup \mathrm{Th}(\mathbf{N})$ has a model. We prove that for every finite subset $\left\{\sigma_{1} \ldots, \sigma_{n}\right\}$ of $\operatorname{Diag}(\mathbf{M}),\left\{\sigma_{1} \ldots, \sigma_{n}\right\} \cup \operatorname{Th}(\mathbf{N})$ has a model. Let $c_{m_{1}}, \ldots, c_{m_{k}}$ be the object constants of the expanded language that occur in $\left\{\sigma_{1} \ldots, \sigma_{n}\right\}$. For every $1 \leq i \leq n$, let $\sigma_{i}^{\prime}$ be the formula obtained from $\sigma_{i}$ by substituting the constants $c_{m_{1}}, \ldots, c_{m_{k}}$ by new variables $\bar{y}=y_{m_{1}}, \ldots, y_{m_{k}}$.

Then we have that $\left\|(\exists \bar{y})\left(\sigma_{1}^{\prime} \wedge \cdots \wedge \sigma_{n}(\bar{y})\right)\right\|_{\mathbf{M}}=1$ and thus, by the assumption of this lemma, since $(\exists \bar{y})\left(\sigma_{1}^{\prime} \wedge \cdots \wedge \sigma_{n}^{\prime}(\bar{y})\right)$ is a pp-sentence, $\|(\exists \bar{y})\left(\sigma_{1}^{\prime} \wedge \cdots \wedge\right.$ $\left.\sigma_{n}(\bar{y})\right) \|_{\mathbf{N}}=1$. Since $\mathbf{N}$ is an $\exists$-witnessed structure, we have a sequence of elements of $\mathrm{N}, \bar{e}=e_{m_{1}}, \ldots, e_{m_{k}}$, such that $\|\left(\left(\sigma_{1}^{\prime} \wedge \cdots \wedge \sigma_{n}(\bar{e}) \|_{\mathbf{N}}=1\right.\right.$. Thus we can conclude that an expansion of $\mathbf{N}$ satisfies $\left\{\sigma_{1} \ldots, \sigma_{n}\right\} \cup \operatorname{Th}(\mathbf{N})$. By $\mathbf{A}$ compactness for satisfiability, there is a $\mathcal{P}$-structure $\mathbf{L}$ that has an expansion which is a model of $\operatorname{Diag}(\mathbf{M}) \cup \operatorname{Th}(\mathbf{N})$.

By Lemma 1, there is a homomorphism $g$ from $\mathbf{M}$ into $\mathbf{L}$. Moreover, since $\mathbf{L}$ is a model of $\operatorname{Th}(\mathbf{N}), \mathbf{L}$ is elementary equivalent to $\mathbf{N}$.

Now we prove an axiomatization theorem for theories closed under homomorphisms and direct products. Recall that a theory $T$ is closed under a class $O$, if the class of its models, $\operatorname{Mod}(T)$, is closed under $O$. And it is say that a theory $T$ is axiomatized by a set of sentences $\Sigma$, if $\operatorname{Mod}(T)=\operatorname{Mod}(\Sigma)$.

Theorem 2. Let $\mathcal{P}$ be a predicate language and $T$ be a consistent theory. Then, $T$ is closed under homomorphisms and direct products if and only if $T$ is axiomatized by a set of positive primitive sentences.

Proof. Let $T_{\vee}$ be the set of finite disjunctions of pp-sentences evaluated positively in every model of $T$ (that is, evaluated with an element of the algebra $a \in A$ such that $a>0$ ), and $T_{p p}$ be the set of pp-sentences of $T_{\vee}$. In the proof we distinguish two parts: 1 ) we show that $T_{\vee}$ axiomatizes $T$, and 2) we show that $T_{p p}$ axiomatizes $T_{\vee}$.

1) $T_{\vee}$ axiomatizes $T$. First notice that $T_{\vee}$ is a nonempty set, for instance $(\exists x)(x \approx x) \in T_{\vee}$, and $T_{\vee}$ is satisfiable because $T$ is a consistent theory. Let $\mathbf{N}$ be a model of $T_{\vee}$, we will show that $\mathbf{N}$ is also a model of $T$. Let $\Gamma$ be the set of all sentences of the form $\neg \delta$, where $\delta$ is a pp-sentence and $\|\delta\|_{\mathbf{N}}<1$. Now we prove that $\Gamma \cup T$ has a model. 
If $\Gamma=\emptyset$ is clear, because $T$ is consistent. If $\Gamma \neq \emptyset$, we have that, for every nonempty subset $\left\{\neg \delta_{1} \ldots, \neg \delta_{n}\right\}$ of $\Gamma,\left\{\neg \delta_{1} \ldots, \neg \delta_{n}\right\} \cup T$ has a model. Otherwise, in every model of $T$ the sentence $\delta_{1} \vee \cdots \vee \delta_{n}$ will be evaluated positively and thus, $\delta_{1} \vee \cdots \vee \delta_{n} \in T_{\vee}$, contradicting the fact that $\mathbf{N}$ is a model of $T_{\vee}$. By $\mathbf{A}-$ compactness for satisfiability, there is a model $\mathbf{M}$ of $\Gamma \cup T$. Then we have that every pp-sentence which is evaluated 1 in $\mathbf{M}$, is also evaluated 1 in $\mathbf{N}$, because $\mathbf{M}$ is a model of $\Gamma$. Then, by Proposition 1, there is a structure $\mathbf{L}$, elementarily equivalent to $\mathbf{N}$, and a homomorphism $g$ from $\mathbf{M}$ into $\mathbf{L}$. Since $T$ is closed under homomorphisms, we can conclude that $\mathbf{N}$ is also a model of $T$. Consequently, $T_{\vee}$ is a set of axioms for $T$.

1) $T_{p p}$ axiomatizes $T_{\vee}$. We show that, for every $\delta_{1} \vee \cdots \vee \delta_{n} \in T_{\vee}$, there is $1 \leq i \leq n$ with $\delta_{i} \in T_{p p}$. Assume, searching for a contradiction, that for every $1 \leq i \leq n$, there is $\mathbf{M}_{i}$ which is a model of $T_{\vee}$ but not of $\delta_{i}$. Consider the direct product $\prod_{1<i<n} \mathbf{M}_{i}$. Since $T_{\vee}$ is closed under direct products, $\prod_{1<i<n} \mathbf{M}_{i}$ is also a model of $T_{\vee}$ and, in particular, of the sentence $\delta_{1} \vee \cdots \vee \delta_{n}$. Then, for some $1 \leq i_{0} \leq n, \prod_{1<i<n} \mathbf{M}_{i}$ is a model of $\delta_{i_{0}}$. Take the $i_{0}$-projection function $i_{0}: \prod_{1 \leq i \leq n} \mathbf{M}_{i} \rightarrow \mathbf{M}_{i_{0}}$. Since $i_{0}$ is a homomorphism, $i_{0}$ preserves pp-formulas, and thus, $\mathbf{M}_{i_{0}}$ is also a model of $\delta_{i_{0}}$, contradicting our original assumption. We can conclude that there is some $1 \leq i \leq n$ with $\delta_{i} \in T_{p p}$. Consequently, $T_{p p}$ is a set of axioms for $T_{\mathrm{V}}$.

Notice that, in the proof of Theorem 2, we have only used finite direct products. Using A-compactness for consequence we can obtain the following corollary of Theorem 2.

Corollary 2. Let $\mathcal{P}$ be a predicate language and $\phi$ be a satisfiable sentence. Then, $\phi$ is equivalent to a pp-sentence if and only if $\phi$ is preserved under homomorphisms and direct products.

\section{Discussion and Future Work}

Can non-classical logic contribute to the analysis of complexity in computer science? We started the paper with the statement of this general question, and in this final section, we would like to comment on how the axiomatization theorem can be regarded as a contribution to provide an answer to this question.

In one of the books of reference in the field [4], model theory is described as algebra+logic. Working in this same framework, and in the line of recent works taking an algebraic approach to valued CSP (see for instance [16] and [17]), we have presented an algebraic characterization of the preservation of ppformulas in terms of direct products and homomorphisms. Theorem 2 tells us that there is a good trade-off between algebra and logic in the fuzzy positiveprimitive fragment. This result allows us also to characterize pp-definability in terms of polymorphisms, that can be defined using homomorphisms and finite direct products.

However, the notion of fuzzy homomorphism traditionally used in the fuzzy literature, do not encompass other notions of polymorphism such as weighted 
or fractional polymorphisms (see for instance [16] or [17]). Further research is needed to study stronger definitions of homomorphism (for example [22], [7] or [9]) and see which are more adequate for the purpose of rephrasing FCSP using homomorphism problems. One of the main characteristics we have to impose to homomorphisms, is that they preserve positive values. Theorem 2 also sheds light to the fact that, if we introduce stronger notions of homomorphisms, we will need to redefine pp-formulas, possibly using a language expanded with constant symbols for the elements of the valued structure, in order to maintain the correspondence between algebra and logic.

The relational structures we have studied are over finite algebras, but we have proven the results both, for finite and for infinite domains, in order to cope with applications on infinite templates. Work in progress includes the generalization of Geiger's Theorem [13] to the fuzzy context, where some important preliminary results were obtained in [22], for locally finite valuation structures. In the classical case, the pp-preservation problem restricted to finite structures was solved by B. Rossman in [23], with some previous results, for instance in [1], in the context of CSP dualities. It would be interesting to prove the corresponding version in the fuzzy context, especially taking into account the improvements recently introduced in [24], with respect to the bounds on the quantifier-rank of the sentences.

\section{Acknowledgements}

The research leading to these results has received funding from RecerCaixa. This project has also received funding from the European Union's Horizon 2020 research and innovation program under the Marie Sklodowska-Curie grant agreement No 689176 (SYSMICS project), and by the projects RASO TIN201571799-C2-1-P, CIMBVAL TIN2017-89758-R, and the grant 2017SGR-172 from the Generalitat de Catalunya.

\section{References}

1. Atserias, A., Dawar A., Kolaitis, Ph. G.: On preservation under homomorphisms and unions of conjunctive queries. J. ACM, 53(2):208-237 (2006)

2. Bellman, R.E., Zadeh, L.A.: Decision-making in a fuzzy environment. Manag. Science, 17:141-164 (1970)

3. Bistarelli, S., Montanari, U., Rossi, F.: Semiring-based constraint satisfaction and optimization. J. ACM, 44(2):201236 (1997)

4. Chang, C.C., Keisler, H.J.: Model Theory. Elsevier Science Publishers (1973)

5. Cintula, P., Hájek, P.: Triangular norm based predicate fuzzy logics. Fuzzy Sets and Systems, 161:311-346 (2010)

6. Cintula, P., Hájek, P., Noguera, C. (eds.): Handbook of Mathematical Fuzzy Logic, volume 37, Studies in Logic, Mathematical Logic and Foundations (2011)

7. Dellunde, P.: Preserving mappings in fuzzy predicate logics. Journal of Logic and Computation, 22(6):1367-1389 (2011). 
8. Dellunde, P.: Applications of ultraproducts: from compactness to fuzzy elementary classes. Logic Journal of the IGPL 22(1):166-180 (2014)

9. Dellunde, P:, García-Cerdaña, A., Noguera, C.: Löwenheim-Skolem theorems for non-classical first-order algebraizable logics. Log. J. IGPL 24(3):321-345 (2016)

10. Dubois, D., Fargier, H., Prade, H.: The calculus of fuzzy restrictions as a basis for flexible constraint satisfaction. In 2nd IEEE Int. Conf. on Fuzzy Systems. IEEE (1993)

11. Esteva, F., Godo, L.: Monoidal t-norm based logic: towards a logic for leftcontinuous t-norms. Fuzzy Sets and Systems, 124:271-288 (2001)

12. Fargier H., Lang, J.: Uncertainty in constraint satisfaction problems: a probabilistic approach. In Proceedings of the European Conference on Symbolic and Quantitative Approaches to Reasoning and Uncertainty, 747:97-104 (1993)

13. Geiger, D.: Closed systems of functions and predicates. Pacific Journal of Mathematics, 27(1) (1968)

14. Hodges, W.: Model Theory. Cambridge (1993)

15. Kolaitis, P. G., Vardi, M.Y.: A Logical Approach to Constraint Satisfaction. In Complexity of Constraints - An Overview of Current Research Themes, LNCS 5250, pages $125-155$ (2008)

16. Kolmogorov, V., Krokhin, A., Rolinek, M.: The Complexity of General-Valued CSPs. In FOCS, pages 1246-1258 (2015)

17. Krokhin, A.A., Zivny, S.: The Complexity of Valued CSPs. The Constraint Satisfaction Problem 2017: 233-266.

18. Meseguer, P., Rossi, F., Schiex, T.: Soft constraints. In Handbook of constraint programming, chapter 9, pages 281-328 (2006).

19. Moura J., Prade, H.: Logical Analysis of Fuzzy Constraint Satisfaction Problems. In 7nd IEEE Int. Conf. on Fuzzy Systems. IEEE (1993)

20. Pini, M.S., Rossi, F., Venable, K.R.: Compact Preference Representation via Fuzzy Constraints in Stable Matching Problems. ADT 2017: 333-338.

21. Rossi, F., Brent, K., Walsh, T.: A Short Introduction to Preferences, Synthesis Lectures on Artificial Intelligence and Machine Learning, Morgan and Claypool Pub. (2011)

22. Horcík, R., Moraschini, T., Vidal, A.: An Algebraic Approach to Valued Constraint Satisfaction. In 26th EACSL Annual Conference on Computer Science Logic, 42:142:20 (2017)

23. Rossman, B.: Homomorphism preservation theorems. J. ACM 55(3): 15:1-15:53 (2008)

24. Rossman, B.: An Improved Homomorphism Preservation Theorem From Lower Bounds in Circuit Complexity. ITCS 2017, 27:1-27.

25. Ruttkay, Z.: Fuzzy constraint satisfaction. In 3rd IEEE Int. Conf. on Fuzzy Systems. IEEE (1994)

26. Schiex, T., Fargier, H., Verfaillie, G.: Valued constraint satisfaction problems: Hard and easy problems. In Proceedings of the Fourteenth International Joint Conference on Artificial Intelligence (IJCAI 95) pages 631-639 (1995)

27. Torra, V.: On Considering Constraints of Different Importance in Fuzzy Constraint Satisfaction Problems. International Journal of Uncertainty, Fuzziness and Knowledge-Based Systems 6(5):489-502 (1998) 